\title{
The effectiveness of a class designed to improve nurses' self-care, relationship with colleagues, and relationship with patients
}

\author{
Melissa Tayarani ${ }^{1}$ and Bonnie Raingruber ${ }^{2 *}$ \\ ${ }^{1}$ Administrative Nurse 2, Patient Care Services, University of California Davis Medical Center, Sacramento, California, USA \\ ${ }^{2}$ Nurse Researcher, Center for Nursing Research, University of California Davis Medical Center, Sacramento, California, USA
}

\begin{abstract}
Purpose: To evaluate the effectiveness of a class designed to improve nurses' self-care, rapport with colleagues, and relationship with patients.

Design/Method: A pre-and post-class survey was used to determine the effectiveness of a 3-day Reigniting the Spirit of Caring (RSC, 2014) course.

Results: The greatest improvement (over a 15\% increase) was seen in four responses: a) I take care of myself at work (59.67\% pre-test compared to $77.77 \%$ post-test-a $18.1 \%$ increase), b) This environment motivates me to grow and develop as a professional (71.86\% pre-test compared to $90.53 \%$ post-test-s $18.67 \%$ increase), c) $\mathrm{My}$ work allows me to put into practice the idea that "caring is the heart of nursing" (76.18\% pre-test compared to $93.04 \%$ post-test-a $16.86 \%$ increase), and d) Healing comes primarily from a relationship with patients (64.5\% pre-test compared to $84.94 \%$ post-test-a $20.44 \%$ increase).
\end{abstract}

Discussion: It is clear that offering a course to improve patient care, worker's self-care, and relationships with other professionals is effective. It may be that each person who attends a Reigniting the Spirit of Caring (RSC, 2014) program acts as a change agent and shares their positive attitude with co-workers.

\section{Introduction}

Caring relationships are the essence of nursing. Self-care, compassionate care for patients, and caring relationships with other professionals are all part of caring [1]. Preserving a culture of care within a hospital results in higher patient satisfaction, enhanced quality of care, greater teamwork, and better nurse retention. One way to foster a culture of caring is to offer continuing education that focuses on the importance of personal responsibility, self-care, and caring relationships with patients and other professionals. However, insufficient evidence exists in the literature that offering such classes helps create a culture of care within academic medical centers [2]. Therefore, we evaluated the effectiveness of a Re-Igniting the Spirit of Caring (RSC) course in our university medical center.

\section{Theoretical underpinning}

Watson's core principles of authentic presence, caring for self and others, maintaining balance, using reflection, cultivating sensitivity to oneself and others, developing trusting relationships, and slowing down to allow space for wonder were the foundations for the ReIgniting the Spirit of Caring course and the survey items that were used to evaluate it $[1,3]$.

\section{Background literature and significance of the study}

Only one article in the literature reported on the effectiveness of continuing nurse education focused on caring and was based on the curriculum, "Reigniting the Spirit of Caring, 2014" from ${ }^{\circ}$ Creative Health Care Management (CHCM). Glembocki and Dunn [2]. surveyed thirty-six registered nurses before and after the 3-day
Reigniting class using the Caring Assessment for the Caregiver tool. Paired t-tests indicated nurse perceptions of caring behaviors increased after the course with the exception of the being-with subscale. The caring behaviors that increased following the class were the ability to convey empathy, act in a non-judgmental manner, spend time with patients, enhance teamwork, and act as a patient advocate [2].

Two studies have used Crew Resource Management (CRM) trainings to increase teamwork in acute care hospitals. A CRM training program was provided to 70 nurses and 88 physicians in a perinatal unit of an academic medical center. Class sizes were limited to 25; lasted 4 hours, included didactic content and role playing activities focused on communication, leadership, and error prevention and were followed by a 2-hour booster course. Twice daily team meetings were also implemented following the training. It was not possible to determine which of these interventions most influenced outcomes. Data was collected prior to the training, after the CRM training, and after 1 year using the Teamwork and Safety Climate subscales of the Safety Attitudes Questionnaire. One year after implementation, significant improvement occurred in both nurses and physician's perceptions of teamwork, although nurses reported lower levels of teamwork than physicians [4]. Kalisch, Xie and Ronis [5] used a CRM leadership and teamwork training lasting one hour that was offered on 3 occasions.

Correspondence to: Bonnie Raingruber, PhD, RN, Nurse Researcher, Center for Nursing Research, University of California Davis Medical Center, Sacramento, California, USA, Tel: 9167347850; E-mail: bjraingruber@ucdavis.edu

Received: February 09, 2017; Accepted: March 02, 2017; Published: March 06 , 2017 
Two hundred forty-two nurses from three acute care hospitals completed the Nursing Teamwork Survey and the MISSARE survey before, immediately after, and two months after the training. Following the intervention teamwork knowledge and satisfaction increased and missed nursing care decreased. Changes were even more significant at the two month period than immediately following the training.

Two studies used reflective practice sessions and measured levels of leadership, caring, and empathy following the seminars. Sands, Stanley and Charon [6] offered weekly narrative training to 19 staff (physicians, nurses, social workers, and child life workers) on a pediatric oncology unit over a 6-week period. Individuals wrote about their clinical experiences and their attachment to patients, read aloud their narratives to other participants, and participated in a facilitated discussion of these experiences. Before and immediately post intervention the Interpersonal Reactivity Index (IPI) was administered. Scores on the perspective taking subscale improved. Goudreau and colleagues [7] used a reflective practice continuing education training to examine personal values, clinical reasoning, and leadership skills of 55 newly graduated nurses working on eight units in two acute care teaching hospitals. Thirty-minute reflective practice sessions included 4-5 nurses (experienced and new nurses) with nurses being asked to attend at least 10 sessions during a 20 week period. A new graduate nurse spoke at each session about a troubling event, a debriefing discussion was held, and reflective journaling was completed that focused on similar situations. Journals were analyzed using a qualitative approach. Results indicated that the reflective practice session enhanced clinical reasoning (ability to gather data, make links among data, and question others), time management, and leadership skills (confidence and ability to talk to other professionals). Participants appreciated having a space and mechanism to process their clinical experiences, to interact with other nurses, and to develop trust.

\section{Purpose}

Additional research regarding the effectiveness of classes designed to improve nurses' self-care, relationship with colleagues, and relationship with patients is needed. The purpose of our survey was to evaluate these caring relationships. Since the Reigniting the Spirit of Caring (RCS, 2014) program from ${ }^{\circ}$ Creative Healthcare Management focuses on these topics we decided to evaluate nurses' perceptions immediately before and after the RCS program.

\section{Design/Method}

A descriptive, cross-sectional, pre and post class survey was used to determine the effectiveness of a 3-day Reigniting the Spirit of Caring program in an academic medical center.

\section{Re-Igniting the Spirit of Caring (RSC) Program Description}

Re-Igniting the Spirit of Caring is a licensed program offered by ${ }^{\bullet}$ Creative Healthcare Management out of Minneapolis, Minnesota. The program is a 3 day experiential program with established objectives and curriculum. The program makes use of adult learning theory, reflection, and story-telling while creating a safe environment for participants to share their experiences. Throughout the course the goal is to get in touch with one's core values, discuss the importance of self-care while caring for others, and to reintroduce humanity into the very human work of healthcare. While this program is designed for the whole team, caregivers and service support staff, we provided it primarily for nurses. The course was taught by two to three certified nurse facilitators each session, which kept participants involved and interested. Multiple facilitators were used to bring diversity to the three days. While all facilitators were trained in the course content, all had unique work experiences and perspectives to share.

\section{Setting}

The program was offered to staff of an academic medical center with 619 licensed inpatient beds. The hospital is a Magnet designated quaternary care center and the only Level 1 Trauma and Pediatric Trauma Center in the northern portion of California. Fifty-two percent (52\%) of patients at this facility are admitted through the emergency room. Of the 33 counties within the service area, 20 are designated as medically underserved. Thirty-seven percent of patients are covered by Medi-Cal, followed by Medicare at 31.5\%, managed care contracts at $26 \%$, and all other insurances at $5.7 \%$. Nurses belong to the California Nurses' Association. A primary care nursing model and an all RN staff is used and with the exception of one inpatient palliative care unit (where Licensed Vocational Nurses are employed). The patient population and the nursing population are diverse as is seen in the Table 1 .

\section{How the facilitators were selected}

Choosing the right facilitators for the program was essential to the program's success. Qualities that are looked for are the ability to clearly articulate feelings and thoughts, and having a current role related to patient care as it gives the facilitator credibility with the participants who work at the bedside. Listening skills are essential as is the ability to engage in healthy relationships, and to speak from the heart. Facilitators are chosen based on these qualities and not by job titles; being in a formal educator role does not guarantee that one would be a good choice to teach this course which requires intuitive facilitation. All of the facilitators were chosen because they live their core values and treat their patients, families and colleagues with true empathy, and deep caring. Once chosen to be a facilitator, there is a training process that must be completed. The new facilitators must attend a weeklong training provided by ${ }^{\circledR}$ Creative Healthcare Management (CHCM) to learn the program; this also allows the $\mathrm{CHCM}$ consultants to evaluate the facilitators. There are many attributes that make a great facilitator including positivity, being supportive, knowing the work culture, having the skills to read people, and maintaining confidentiality. It cannot be understated that choosing a facilitator that embraces and lives these qualities, along with the great curriculum, contributes to providing a rich, enduring experience for the participants.

\section{How the program was organized}

Re-Igniting the Spirit of Caring was added to the catalog of courses available to nurses at our institution shortly after Primary Nursing was instituted as a care model. Both programs are closely aligned with similar values and both keep patients at the center of care decisions. Many nurses have said through their evaluations that RSC should be mandatory for all nurses, physicians, and interdisciplinary staff,

Table 1. Hospital and community wide demographic information.

\begin{tabular}{|l|c|c|c|}
\hline Ethnicity & $\begin{array}{c}\text { Ethnicity of } \\
\text { community served }\end{array}$ & $\begin{array}{c}\text { Patient } \\
\text { ethnicity }\end{array}$ & $\begin{array}{c}\text { Nursing staff } \\
\text { ethnicity }\end{array}$ \\
\hline White & $57 \%$ & $34.5 \%$ & $57 \%$ \\
\hline Black & $10 \%$ & $9.3 \%$ & $4 \%$ \\
\hline Hispanic & $21 \%$ & $12.2 \%$ & $7 \%$ \\
\hline Asian \& Pacific Islander & $14 \%$ & $5 \%$ & $30 \%$ \\
\hline Native American & $1 \%$ & $0.1 \%$ & $1 \%$ \\
\hline Multi race & $2.7 \%$ & $38.9 \%$ & $6 \%$ \\
\hline Not specified & & & $<1 \%$ \\
\hline
\end{tabular}


but the course is more meaningful if the participant has chosen to be there and not forced to attend. Interested individuals can access the class information through the nursing education website where there is a course description and details for the class. We also distributed flyers with class information and dates throughout the inpatient units. Presentations about the program were made at the Clinical Nurse III all here days, the Administrative Nurse meetings, and to new graduate orientations knowing these nurses could take the information back to their departments.

Since the course itself is a licensed program from ${ }^{\circ}$ Creative Healthcare Management, the company provides journals for the course participants. Over the course of the three days, self-care is valued, discussed, and prioritized which is why every day of the workshop was catered for breakfast and lunch. Providing a site outside of the hospital contributed to a sense of calm and caring, encouraging attendees to experience three days away from work. The hospital administration believes this program is important and understands how difficult working in healthcare can be which is why the first time the participants attend the class their time is paid ( 24 hours total over 3 days). Many nurses select to attend the course more than once and there is professional leave time that they can use for the second and third time they wish to attend.

\section{Survey development; Ethical protections; Data Collection and Data Analysis}

Two authors developed the survey questions based on a review of the literature and content covered in the RSC class. Each question was identified as belonging to a sub category of relationships with patients, relationships with colleagues or self-care. All course facilitators evaluated the survey for face validity. The accuracy of data entry was validated by two light duty nurses. The Institutional Review Board determined the survey was exempt. Surveys were completed during the first 10 minutes of the Reigniting the Spirit class and during the last 10 minutes on day 3 . All responses were anonymous and collected without associated demographic information. The results were analyzed using descriptive statistics and by reporting percentile responses.

\section{Participant demographics}

Participant demographic information was not collected before or after the class when the survey responses were collected. This was done to maintain participant anonymity. Rather a list of participant names was given to personnel and composite demographic information (that was not linked to survey responses) was obtained. Seventy-three individuals participated including 65 (89\%) nurses, 2 (3\%) technicians, and $6(8 \%)$ respiratory therapists. Twenty individuals $(27 \%)$ were between the ages of 18 and 29, 19 (26\%) were between 30 and 39, 24 (33\%) were between 40 and 49 while $10(14 \%)$ were 50 or older. Seven (10\%) individuals were Asian, $15(20 \%)$ as Filipino, 10 (14\%) as Latino/ Chicano, 6 (8\%) as European, $2(3 \%)$ as African American, and 33 $(45 \%)$ as White. Ten participants (14\%) were male while $63(86 \%)$ were female. None of the employees had taken the course previously.

\section{Results}

Table 2, the pre education survey, and Table 3, the post education survey, include both pre and post class responses and percentages for each question on the survey. There was improvement in the expected direction for all questions when one looks at the "strongly agree" column in the pre and post tables except for the question "My colleagues offer to help me when I'm falling behind" (28.57\% strongly agreed in the pre-test while $28.37 \%$ strongly agreed in the post-test). On that question the pre and post course percentages were close.

When comparing the strongly agree responses thirteen questions showed the greatest improvement (over a 15\% increase) including: a) My job allows me to make a difference in people's lives (a $19.74 \%$ increase), b) I am able to have difficult conversations (a $24.09 \%$ increase), c) The environment at my hospital motivates me to grow and develop as a professional (a $25.21 \%$ increase), d) I take responsibility for my professional development (a $17.31 \%$ increase), e) I feel supported by my colleagues (a $18.46 \%$ increase), f) There is a lot of teamwork at my hospital (a 20.79\% increase), g) I work in one of the best hospitals in the area (a 27.65\% increase), h) My work allows me to put into practice the idea that caring is the heart of nursing (a 22.62\% increase), i) Providing excellent patient care is the most important part of my job (a $16.15 \%$ increase), j) My positivity impacts others in my work environment (a $27.49 \%$ increase), k) I offer to help my colleagues when they need help (a $15.85 \%$ increase), 1) Healing comes primarily from the relationship with patients (a $21.51 \%$ increase), and $\mathrm{m}$ ) I feel privileged to work at my hospital (a $18.71 \%$ improvement).

When adding the "agree" and "strongly agree" columns most responses changed by $10 \%$ or more in the expected direction (indicating improvement). Two exceptions where there were minor decreases were the following questions: a) I take responsibility for my professional development (100\% either strongly agreed or agreed in the pre-test compared to $98.64 \%$ in the post-test), and b) I am proud of what I do for a living ( $100 \%$ either strongly agreed or agreed in the pretest compared to $98.64 \%$ in the post-test). For those two questions the pre and post course percentages were close.

When adding the strongly agree and agree responses the four questions that showed the greatest improvement (over a 15\% increase) included: a) I take care of myself at work (59.67\% pre-test compared to $77.77 \%$ post-test-a $18.1 \%$ increase), b) The environment at my hospital motivates me to grow and develop as a professional (71.86\% pre-test compared to $90.53 \%$ post-test-a $18.67 \%$ increase), c) My work allows me to put into practice the idea that "caring is the heart of nursing" (76.18\% pre-test compared to $93.04 \%$ post-test-a $16.86 \%$ increase), and d) Healing comes primarily from the relationship with patients $(64.5 \%$ pre-test compared to $84.94 \%$ post-test-a $20.44 \%$ increase).

\section{Limitations/Suggestions for Future Research}

As this was a self-report survey we did not evaluate employee retention rates, patient outcomes, actual self-care behaviors, or standardized measures of teamwork or caring. The survey was conducted at only one academic medical center and included individuals from a variety of disciplines. Future research should evaluate courses offered in multiple hospitals and varied types of hospitals. Due to funding limitations and the fact that we did not collect demographic information when the survey data was obtained we did not run correlations between demographic data and survey responses. We also did not conduct long term follow up surveys to see if changes in attitude were sustained. Funding did not allow for providing a booster course although nurses were allowed to re-take the course using paid professional leave if they selected to do so. Not all attendees responded to all questions, although the most questions that were skipped were three. Future research should include at least 30 individuals from a given profession in each class. For practical reasons we included 65 nurses, 2 technicians, and 6 respiratory therapists because nursing administration had recommended that the class be multidisciplinary and these individuals asked to attend. Other researchers should explore 
Table 2. Pre-education survey results.

\begin{tabular}{|c|c|c|c|c|c|c|c|}
\hline Question & Sub category & Strongly Agree & Agree & Don't Know & Disagree & $\begin{array}{l}\text { Strongly } \\
\text { Disagree }\end{array}$ & Total Subjects \\
\hline \multirow{2}{*}{$\begin{array}{l}\text { My job allows me to make a difference in people's } \\
\text { lives. }\end{array}$} & \multirow{2}{*}{ Relation-ship with patients } & 41 & 17 & 3 & 3 & \multirow[t]{2}{*}{0} & \multirow[t]{2}{*}{64} \\
\hline & & $64.06 \%$ & $26.56 \%$ & $4.68 \%$ & $4.68 \%$ & & \\
\hline \multirow[t]{2}{*}{ I am able to have difficult conversations. } & \multirow{2}{*}{$\begin{array}{l}\text { Relation-ship with } \\
\text { colleagues }\end{array}$} & 9 & 38 & 9 & 7 & \multirow[t]{2}{*}{0} & \multirow[t]{2}{*}{63} \\
\hline & & $14.28 \%$ & $60.31 \%$ & $14.28 \%$ & $11.11 \%$ & & \\
\hline \multirow{2}{*}{$\begin{array}{l}\text { Several times a week I find myself thinking about } \\
\text { things that happened at work that bothered me. }\end{array}$} & \multirow[t]{2}{*}{ Self-care } & 14 & 35 & 2 & 10 & 2 & \multirow[t]{2}{*}{63} \\
\hline & & $22.22 \%$ & $55.55 \%$ & $3.17 \%$ & $15.87 \%$ & $3.17 \%$ & \\
\hline \multirow[t]{2}{*}{ I take care of myself at work. } & \multirow[t]{2}{*}{ Self-care } & 8 & 29 & 6 & 16 & 3 & \multirow[t]{2}{*}{62} \\
\hline & & $12.90 \%$ & $46.77 \%$ & $9.67 \%$ & $25.80 \%$ & $4.83 \%$ & \\
\hline \multirow{2}{*}{$\begin{array}{l}\text { I include my patients when planning the care, I } \\
\text { provide. }\end{array}$} & \multirow[t]{2}{*}{ Relation-ship with patients } & 28 & 29 & 4 & 1 & \multirow[t]{2}{*}{0} & 62 \\
\hline & & $45.16 \%$ & $46.77 \%$ & $6.45 \%$ & $1.61 \%$ & & \\
\hline The environment at my hospital motivates me to & Self-care & 15 & 31 & 9 & 9 & 0 & 64 \\
\hline grow and develop as a professional. & & $23.43 \%$ & $48.43 \%$ & $14.06 \%$ & $14.06 \%$ & & \\
\hline I am considering leaving my profession. & Relation-ship with & 1 & 7 & 5 & 20 & 30 & 63 \\
\hline & & $1.58 \%$ & $11.11 \%$ & $7.93 \%$ & $31.74 \%$ & $47.61 \%$ & \\
\hline I take responsibility for my professional & Self-care & 28 & 36 & 0 & 0 & 0 & 64 \\
\hline development. & & $43.75 \%$ & $56.25 \%$ & & & & \\
\hline I feel supported by my colleagues. & Relation-ship with & 15 & 37 & 6 & 6 & 0 & 64 \\
\hline & colleagues & $23.43 \%$ & 57.81 & 9.37 & 9.37 & & \\
\hline I respect the people I work with. & Relation-ship with & 31 & 30 & 0 & 3 & 0 & 64 \\
\hline & & $48.43 \%$ & $46.87 \%$ & & $4.68 \%$ & & \\
\hline I am considering leaving my institution. & Relation-ship with & 1 & 4 & 10 & 20 & 27 & 62 \\
\hline & colleagues & $1.61 \%$ & $6.45 \%$ & $16.10 \%$ & $32.25 \%$ & $43.54 \%$ & \\
\hline There is a lot of teamwork at my institution. & Relation-ship with & 15 & 40 & 3 & 4 & 1 & 63 \\
\hline & colleagues & $23.80 \%$ & $63.49 \%$ & $4.76 \%$ & $6.34 \%$ & $1.58 \%$ & \\
\hline I work in one of the best hospitals in the area. & Relation-ship with & 26 & 29 & 6 & 1 & 1 & 63 \\
\hline & colleagues & $41.26 \%$ & $46.08 \%$ & $9.52 \%$ & $1.58 \%$ & $1.58 \%$ & \\
\hline I express appreciation to my co-workers on a & Relation-ship with & 28 & 32 & 2 & 2 & 0 & 64 \\
\hline regular basis. & colleagues & $43.75 \%$ & $50 \%$ & $3.12 \%$ & $3.12 \%$ & & \\
\hline My work allows me to put into practice the idea & Relation-ship with patients & 19 & 29 & 11 & 4 & 0 & 63 \\
\hline that "caring is the heart of nursing". & & $30.15 \%$ & $46.03 \%$ & $17.46 \%$ & $6.34 \%$ & & \\
\hline Providing excellent patient care is the most & Relation-ship with patients & 35 & 25 & 2 & 0 & 0 & 62 \\
\hline important part of my job. & & $56.45 \%$ & $40.32 \%$ & $3.22 \%$ & & & \\
\hline I am so busy I can't devote sufficient time to my & Relation-ship with patients & 8 & 16 & 6 & 25 & 7 & 62 \\
\hline & & $12.90 \%$ & $25.80 \%$ & $9.67 \%$ & $40.32 \%$ & $11.29 \%$ & \\
\hline My positivity impacts others in my work & Relation-ship with & 17 & 37 & 10 & 0 & 0 & 64 \\
\hline environment. & colleagues & $26.56 \%$ & 57.81 & 15.62 & & & \\
\hline I offer to help my colleagues when they need help. & Relation-ship with & 27 & 35 & 1 & 0 & 0 & 63 \\
\hline & colleagues & $42.85 \%$ & $55.55 \%$ & $1.58 \%$ & & & \\
\hline My colleagues offer to help me when I'm falling & Relation-ship with & 18 & 35 & 5 & 5 & 0 & 63 \\
\hline & & $28.57 \%$ & $55.55 \%$ & $7.93 \%$ & $7.93 \%$ & & \\
\hline I am proud of what I do for a living. & Self-care & 44 & 20 & 0 & 0 & 0 & 64 \\
\hline & & $68.75 \%$ & $31.25 \%$ & & & & \\
\hline My schedule is so busy I don't have enough time & Self-care & 5 & 24 & 4 & 25 & 6 & 64 \\
\hline left to take care of myself. & & $7.81 \%$ & $37.50 \%$ & $6.25 \%$ & $39.06 \%$ & $9.37 \%$ & \\
\hline My job is not what I expected it to be. & Relation-ship with & 5 & 10 & 8 & 32 & 7 & 64 \\
\hline & colleagues & $7.81 \%$ & $15.62 \%$ & $12.50 \%$ & $50 \%$ & $14.06 \%$ & \\
\hline I think of my job as a professional career. & Self-care & 44 & 18 & 1 & 1 & 0 & 64 \\
\hline & & $68.75 \%$ & $28.12 \%$ & $1.56 \%$ & $1.56 \%$ & & \\
\hline Healing comes primarily from the relationship & Relation-ship with patients & 13 & 27 & 16 & 6 & 0 & 62 \\
\hline with the patients. & & $20.96 \%$ & $43.54 \%$ & $25.80 \%$ & $9.67 \%$ & & \\
\hline If I were 18 again I would select another career. & Self-care & 4 & 4 & 10 & 22 & 24 & 64 \\
\hline & & $6.25 \%$ & $6.25 \%$ & $15.62 \%$ & $34.37 \%$ & $37.50 \%$ & \\
\hline I look for ways to motivate the people I work with. & Relation-ship with & 12 & 38 & 8 & 6 & 0 & 64 \\
\hline & colleagues & $18.75 \%$ & $59.37 \%$ & $12.50 \%$ & $9.37 \%$ & & \\
\hline I feel privileged to work at my hospital. & Relation-ship with & 33 & 24 & 7 & 0 & 0 & 64 \\
\hline & colleagues & $51.56 \%$ & $37.50 \%$ & $10.93 \%$ & & & \\
\hline
\end{tabular}


Table 3. Post education survey results.

\begin{tabular}{|c|c|c|c|c|c|c|c|}
\hline Question & Sub category & $\begin{array}{l}\text { Strongly } \\
\text { Agree }\end{array}$ & Agree & Don't Know & Disagree & $\begin{array}{l}\text { Strongly } \\
\text { Disagree }\end{array}$ & $\begin{array}{l}\text { Total } \\
\text { Subjects }\end{array}$ \\
\hline \multirow[t]{2}{*}{ My job allows me to make a difference in people's lives. } & \multirow[t]{2}{*}{ Relation-ship with patients } & 62 & 12 & \multirow[t]{2}{*}{0} & \multirow[t]{2}{*}{0} & \multirow[t]{2}{*}{0} & \multirow[t]{2}{*}{74} \\
\hline & & $83.78 \%$ & $16.21 \%$ & & & & \\
\hline \multirow[t]{2}{*}{ I can have difficult conversations. } & \multirow[t]{2}{*}{ Relation-ship with colleagues } & 21 & 44 & 5 & 4 & & \multirow[t]{2}{*}{74} \\
\hline & & $28.37 \%$ & $59.45 \%$ & $6.75 \%$ & $5.40 \%$ & & \\
\hline \multirow{2}{*}{$\begin{array}{l}\text { Several times a week I find myself thinking about things } \\
\text { that happened at work that bothered me. }\end{array}$} & \multirow[t]{2}{*}{ Self-care } & 8 & 39 & 5 & 17 & 3 & \multirow[t]{2}{*}{72} \\
\hline & & $11.11 \%$ & $54.16 \%$ & $6.94 \%$ & $23.61 \%$ & $4.16 \%$ & \\
\hline \multirow[t]{2}{*}{ I take care of myself at work. } & \multirow[t]{2}{*}{ Self-care } & 11 & 45 & 7 & 7 & 2 & \multirow[t]{2}{*}{72} \\
\hline & & $15.27 \%$ & $62.50 \%$ & $9.72 \%$ & $9.72 \%$ & $2.77 \%$ & \\
\hline \multirow[t]{2}{*}{ I include my patients when planning the care, I provide. } & \multirow[t]{2}{*}{ Relation-ship with patients } & 39 & 31 & 0 & 1 & 0 & 71 \\
\hline & & $54.92 \%$ & $43.66 \%$ & & $1.40 \%$ & & \\
\hline The environment at my hospital motivates me to grow & Self-care & 36 & 31 & 5 & 7 & 0 & 74 \\
\hline and develop as a professional. & & $48.64 \%$ & 41.89 & $6.75 \%$ & $2.70 \%$ & & \\
\hline I am considering leaving my profession. & Relation-ship with colleagues & 0 & 2 & 7 & 20 & 45 & 74 \\
\hline & & & $2.70 \%$ & $9.45 \%$ & $27.02 \%$ & 60.81 & \\
\hline I take responsibility for my professional development. & Self-care & 45 & 28 & 1 & 1 & 0 & 74 \\
\hline & & $60.81 \%$ & $37.83 \%$ & $1.35 \%$ & $1.35 \%$ & & \\
\hline I feel supported by my colleagues. & Relation-ship with colleagues & 31 & 39 & 3 & 1 & 0 & 74 \\
\hline & & 41.89 & $52.70 \%$ & $4.05 \%$ & $1.35 \%$ & & \\
\hline I respect the people I work with. & Relation-ship with colleagues & 45 & 27 & 2 & 0 & 0 & 74 \\
\hline & & $60.81 \%$ & $36.48 \%$ & $2.70 \%$ & & & \\
\hline I am considering leaving my institution. & Relation-ship with colleagues & 0 & 5 & 7 & 17 & 44 & 73 \\
\hline & & & $6.84 \%$ & $9.58 \%$ & $23.28 \%$ & $60.27 \%$ & \\
\hline There is a lot of teamwork at my institution. & Relation-ship with colleagues & 33 & 38 & 2 & 1 & 0 & 74 \\
\hline & & $44.59 \%$ & $51.35 \%$ & $2.70 \%$ & $1.35 \%$ & & \\
\hline I work in one of the best hospitals in the area. & Relation-ship with colleagues & 51 & 22 & 1 & 0 & 0 & 74 \\
\hline & & $68.91 \%$ & $29.72 \%$ & $1.35 \%$ & & & \\
\hline I express appreciation to my co-workers on a regular & Relation-ship with colleagues & 35 & 35 & 3 & 1 & 0 & 74 \\
\hline basis. & & $47.29 \%$ & $47.29 \%$ & $4.05 \%$ & $1.35 \%$ & & \\
\hline My work allows me to put into practice the idea that & Relation-ship with patients & 38 & 29 & 4 & 1 & 0 & 72 \\
\hline "caring is the heart of nursing". & & $52.77 \%$ & $40.27 \%$ & $5.40 \%$ & 1.38 & & \\
\hline Providing excellent patient care is the most important part & Relation-ship with patients & 53 & 20 & 0 & 0 & 0 & 73 \\
\hline of my job. & & $72.60 \%$ & $27.39 \%$ & & & & \\
\hline I am so busy I can’t devote sufficient time to my patients. & Relation-ship with patients & 3 & 23 & 8 & 31 & 8 & 73 \\
\hline & & $4.10 \%$ & $31.50 \%$ & $10.95 \%$ & $42.46 \%$ & $10.95 \%$ & \\
\hline My positivity impacts others in my work environment. & Relation-ship with colleagues & 40 & 32 & 1 & 1 & & 74 \\
\hline & & $54.05 \%$ & $43.24 \%$ & $1.35 \%$ & $1.35 \%$ & & \\
\hline I offer to help my colleagues when they need help. & Relation-ship with colleagues & 45 & 27 & 1 & 0 & 0 & 73 \\
\hline & & $61.64 \%$ & $36.98 \%$ & $1.36 \%$ & & & \\
\hline My colleagues offer to help me when I'm falling behind. & Relation-ship with colleagues & 21 & 47 & 2 & 4 & 0 & 74 \\
\hline & & $28.37 \%$ & $63.51 \%$ & $2.70 \%$ & $5.40 \%$ & & \\
\hline I am proud of what I do for a living. & Self-care & 60 & 13 & 0 & 1 & 0 & 74 \\
\hline & & 81.08 & $17.56 \%$ & & $1.35 \%$ & & \\
\hline My schedule is so busy I don't have enough time left to & Self-care & 5 & 18 & 12 & 27 & 10 & 72 \\
\hline take care of myself. & & $6.94 \%$ & $24.32 \%$ & 16.66 & $37.50 \%$ & $13.88 \%$ & \\
\hline My job is not what I expected it to be. & Relation-ship with colleagues & 4 & 10 & 6 & 37 & 17 & 74 \\
\hline & & $5.40 \%$ & 13.51 & $8.10 \%$ & $50 \%$ & $22.90 \%$ & \\
\hline I think of my job as a professional career. & Self-care & 59 & 12 & 2 & 0 & 0 & 73 \\
\hline & & $80.82 \%$ & $16.44 \%$ & $2.74 \%$ & & & \\
\hline Healing comes primarily from the relationship with the & Relation-ship with patients & 31 & 31 & 8 & 3 & 0 & 73 \\
\hline patients. & & $42.47 \%$ & $42.47 \%$ & $10.96 \%$ & $4.11 \%$ & & \\
\hline If I were 18 again I would select another career. & Self-care & 3 & 1 & 8 & 22 & 40 & 74 \\
\hline & & $4.05 \%$ & $1.35 \%$ & $10.81 \%$ & $29.73 \%$ & $54.05 \%$ & \\
\hline I look for ways to motivate the people I work with. & Relation-ship with colleagues & 23 & 40 & 9 & 2 & 0 & 74 \\
\hline & & $31.08 \%$ & $54.05 \%$ & $12.16 \%$ & $2.70 \%$ & & \\
\hline I feel privileged to work at my hospital. & Relation-ship with colleagues & 52 & 21 & 1 & 0 & 0 & 74 \\
\hline & & $70.27 \%$ & $28.38 \%$ & $1.35 \%$ & & & \\
\hline
\end{tabular}


what affect changing the facilitator has given that standardized training and course content is provided by ${ }^{\odot}$ Creative Healthcare Management.

\section{Discussion}

Nurses' responses documented an improvement in the culture of care (compassionate patient care, self-care, and caring relationships with other professionals) following the Re-Igniting the Spirit of Caring program. Following the course responses also indicated improvements in teamwork and intent to pursue future professional development. It is likely that the three days of interactive and supportive time, which the nurses experienced during the RSC class, contributed to a renewed intent to focus on self-care, to treat co-workers with compassion, and to re-commit to providing excellent patient care. Having time away from patient care, the freedom to share stories from practice, and talk about concepts that touch on the very heart of nursing was rejuvenating for nurses. Nurses had time to reflect on and reignite the spirit of caring within their practice. The class provided the space to enjoy and engage in positive interactions with other nurses, to experience an environment where it was safe to share challenging events, and to slow down and remember the reasons they decided to become a nurse in the first place.

Our findings are similar to results from other author's research. Glembocki and Dunn [2] reported increases in nurses' perceptions of caring following a Reigniting the Spirit of Caring course. Several authors also found teamwork increased following educational sessions. Sands and colleagues [6] described increases in teamwork after narrative training; Budin and colleagues [4] reported increases in teamwork following Crew Resource Management training; and Kalisch, Xie, and Ronis [5] found teamwork increased after role-play training. No studies were located which reported findings that were contrary to our results.

\section{Implications for clinical practice, management, and future research}

Interventions designed to improve the health of nurses are valuable and yield dividends in terms of more nurturing and empowering relationships with patients. Self-care training for nurses and improved relationships with colleagues contributes to improved teamwork, and an overall culture of caring within hospital settings. It is clear that hospital administrators benefit by offering courses that are designed to improve patient care, worker's self-care, and relationships with other professionals. It may be that each person who attends a Reigniting the Spirit of Caring acts as a change agent and shares their positive attitude with co-workers. Future research should evaluate that possibility by surveying attendees and their co-workers at several pre and post education periods. It would also be helpful to obtain several post education evaluations to gain a sense of how long the course results influence practice. Since Reigniting the Spirit classes that include only employees from a given unit are planned at this hospital at a future date, qualitative evaluations will be used to determine if a shift in overall unit dynamics occurs following the course. Future studies should also examine whether the class is more or less effective based on years of experience and job classification. Hard outcome measures such as standardized measures of teamwork, standardized measures of caring, employee retention rates, and attendance at employer offered monthly self-care offerings (Yoga, walking club, Tai Chi classes etc.) should also be evaluated.

\section{Acknowledgement}

Thank you to Guillem Lacson, BSN, RN and Jenilyn Bactol BSN, $\mathrm{RN}$ for verifying the accuracy of the data in Tables 1 and 2.

\section{References}

1. Watson J (2006) Caring theory as an ethical guide to administrative and clinical practices. Nurs Adm Q 30: 48-55. [Crossref]

2. Glembocki MM, Dunn KS (2010) Building an organizational culture of caring: Caring perceptions enhanced with education. J Contin Educ Nurs 41: 565-570. [Crossref]

3. Watson J (2008) Nursing: the philosophy and science of caring. Boulder: University Press of Colorado.

4. Budin WC, Gennaro S, O'Connor C, Contratti F (2014) Sustainability of improvements in perinatal teamwork and safety climate. J Nurs Care Qual 29: 363-370. [Crossref]

5. Kalisch BJ, Xie B, Ronis DL (2013) Train-the-trainer intervention to increase nursing teamwork and decrease missed nursing care in acute care patient units. Nurs Res 62: 405-413. [Crossref]

6. Sands SA, Stanley P, Charon R (2008) Pediatric narrative oncology: Interprofessiona training to promote empathy, build teams and prevent burnout. J Support Oncol 6: 307-312. [Crossref]

7. Goudreau J, Pepin J, Larue C, Dubois S, Descoteaux R, et al. (2015) A competencybased approach to nurses' continuing education for clinical reasoning and leadership through reflective practice in a care situation. Nurse Educ Pract 15: 572-578. [Crossref]

Copyright: (C2017 Tayarani M. This is an open-access article distributed under the terms of the Creative Commons Attribution License, which permits unrestricted use, distribution, and reproduction in any medium, provided the original author and source are credited. 